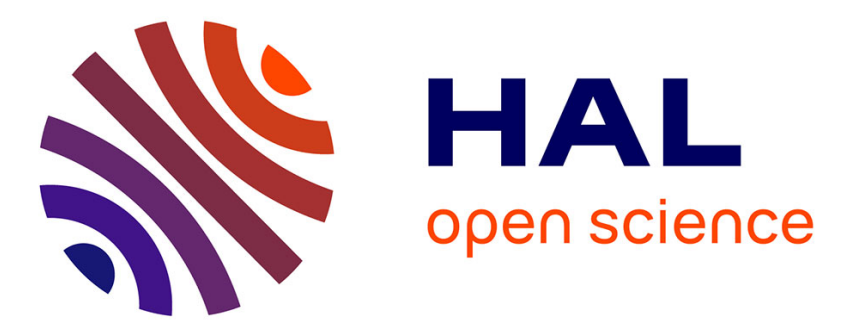

\title{
Performance study of nonlinearities blind correction in wideband receivers
}

Raphael Vansebrouck, Chadi Jabbour, Patricia Desgreys, Olivier Jamin, van Tam Nguyen

\section{> To cite this version:}

Raphael Vansebrouck, Chadi Jabbour, Patricia Desgreys, Olivier Jamin, van Tam Nguyen. Performance study of nonlinearities blind correction in wideband receivers. ICECS, Dec 2014, Marseille, France. 10.1109/ICECS.2014.7049990 . hal-01225463

\section{HAL Id: hal-01225463 \\ https://hal.science/hal-01225463}

Submitted on 6 Nov 2015

HAL is a multi-disciplinary open access archive for the deposit and dissemination of scientific research documents, whether they are published or not. The documents may come from teaching and research institutions in France or abroad, or from public or private research centers.
L'archive ouverte pluridisciplinaire HAL, est destinée au dépôt et à la diffusion de documents scientifiques de niveau recherche, publiés ou non, émanant des établissements d'enseignement et de recherche français ou étrangers, des laboratoires publics ou privés. 


\title{
Performance study of nonlinearities blind correction in wideband receivers
}

\author{
Raphael Vansebrouck ${ }^{* \dagger}$, Chadi Jabbour *, Patricia Desgreys *, Olivier Jamin ${ }^{\dagger}$, and Van-Tam Nguyen* ${ }^{*}$ \\ *Institut TELECOM-TELECOM ParisTech, LTCI-CNRS-UMR 5141, France \\ ${ }^{\dagger}$ NXP Semiconductors, Caen, France \\ $\ddagger$ Department of EECS, University of California at Berkeley, California, 94720, USA \\ Email: raphael.vansebrouck@nxp.com
}

\begin{abstract}
This paper studies a post-distortion correction technique applied to a nonlinear receiver. The employed technique relies on the assumption that the input signal is bandlimited and uses the signal spreading in the free energy band in order to perform the estimation. The study focuses on three points: the input distortion level impact, filter complexity and quantization. A good sizing of these key parameters is mandatory in order to have an optimized CMOS implementation.
\end{abstract}

\section{INTRODUCTION}

Nowadays, digital linearization is becoming a very hot topic. With the growth of digital system and shrinking of CMOS technology, digital signal processing is becoming more and more suitable for this issue. Digital techniques like predistortions [1] are already widely employed in transmitters for power amplifiers (PA) [2] in order to improve their efficiency [3] and avoid polluting adjacent channels with intermodulations terms. Digital post-distortion [4] is a recent and attractive approach to fight against nonlinear systems at the receiver side. It can be used to complete a pre-distortion at the transmitter [5], to correct only the receiver part or it can be applied on its own to correct both the transmitter and the receiver nonlinearities.

Several approaches have been proposed in the literature to implement post-distortion corrections. It can be performed after the demodulation similarly to blind channel estimation for equalization. It uses the symbols statistic and tools like high order statistic (HOS). These techniques can be fully blind [6] or aided [7]. For pre-demodulation corrections, one approach consists in adding a low resolution ADC in parallel with an attenuator to avoid the clipping on the strong carriers [8]. A second method uses an interpolation method based on polyphase decomposition to reconstruct the clipped part and thus improve the linearity [9]. Another interesting approach takes advantage of the property of nonlinear systems to spread the spectrum. Actually, if a frequency band at the input of the nonlinear system is free, distortions will appear in this band due to spectrum spreading. An algorithm will seek coefficients for the correction to restrict the output band to the original input band [10][11][12].

This paper provides a system-level framework for the design of blind post-distortion techniques for band-limited signal, and the evaluation of their fundamental limits. Indeed, existing papers usually focus on the algorithm design, but do not refer to the limitations induced by realistic usercase and practical implementation. This paper provides a view on the limitations of these correction systems, and therefore provides an help for this design. Limitations are demonstrated using the algorithm presented in [12]. The aforementioned paper demonstrates the technique for a unique case with a white noise filtered signal of width 0.3 times the sampling frequency, noted $f_{s}$, and an input SFDR of $60 \mathrm{~dB}$. This paper extends the study, results are shown for several configurations of input signal occupancy and input SFDR. The paper is organized as follows. Section 2 describes the employed blind post-distortion method. Section 3 studies and analyzes, using analytical calculations and systemlevel simulations, the impact of several parameters such as, distortion level impact, filter complexity and quantization. In the last section, concluding remarks are drawn.

\section{PRESENTATION: NONLINEAR CORRECTION SYSTEM STUDIED}

The employed correction system is shown in Fig. 1 [12]. The estimation part is located on the top of the figure and the correction part is located on its bottom. Suppose a weakly nonlinear system, $y(n)=G x(n)+d(n)$, where $G$ represents the linear gain and $d(n)$ the distortions. If $d(n)$ is sufficiently small then $y(n)$ can be approximated by $G x(n)$. Therefore if the nonlinear system has been estimated, an estimation of $d(n)$ can be calculated using $y(n)$. Then, the estimated distortion, $\hat{d}(n)$, is subtracted from $y(n)$. Hence a linearized output $y_{c}(n)$ can be obtained. We will consider here a polynomial model for the following. To construct the estimated distortion, each nonlinear order is estimated individually and then added. The $k^{t h}$ nonlinear component is calculated by $y(n)$ to the power $k$ (pictured by the block " $p=k$ " in Fig. 1) and multiply it by the polynomial coefficient $\alpha_{k}$. The estimation of the coefficients $\alpha$ is done with the NLMS (Normalized Least Mean Squares) algorithm [13] thanks to the signal spreading property with the band-limited input signal hypothesis. The band-limited assumption on $x(n)$ allows to locate a free-energy frequency band where harmonic distortions and intermodulation terms will fall at the output of the nonlinear system. The NLMS algorithm estimates the coefficients by minimizing the cost function $J=E\left[\|e(n)\|^{2}\right]$, where $e(n)$ is the error between the true $(\tilde{y}(n))$ and the estimated distortion $(\tilde{\hat{e}}(n))$ in the free frequency band. This frequency band is recovered by a filter, pictured by $f(n)$ in Fig. 1.

The NLMS is expressed as follows:

$$
\tilde{\alpha}_{p}(n+1)=\tilde{\alpha}_{p}(n)+\mu \frac{y^{p}(n) e(n)}{\sum_{p=1}^{P}\left\|y^{p}(n)\right\|_{2}^{2}},
$$


where $\tilde{\alpha}_{p}(n)$ is the polynomial coefficient of order $p, P$ is the maximum order considered for the correction and $\mu$ is the convergence step. The choice of the NLMS algorithm over the LMS is motivated by a better stability regardless of the input signal statistic and a possible faster convergence compared to the LMS.

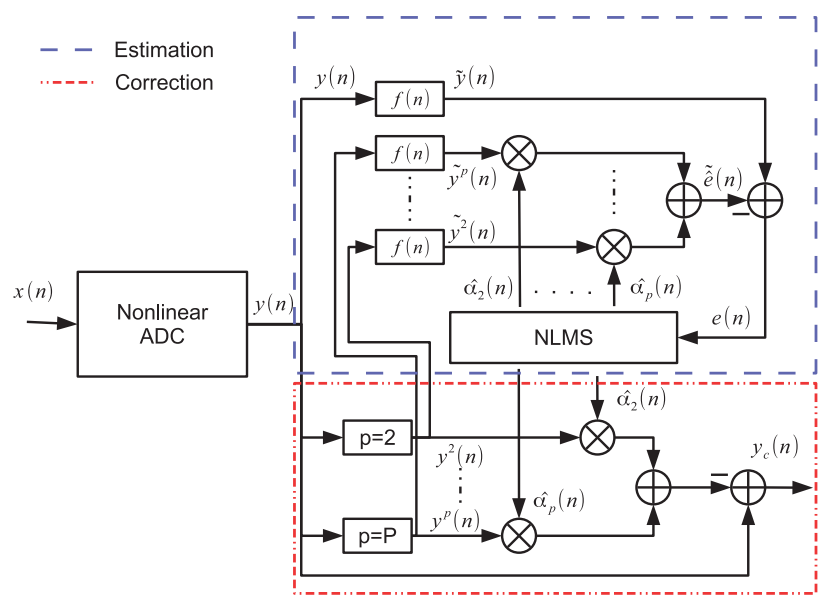

Fig. 1. Estimation and correction scheme

\section{SySTEM PERFORMANCE}

\section{A. Simulation parameters}

As said earlier, the considered system is a nonlinear receiver. In order to limit the calculus length, the nonlinear model is restricted to a third order polynomial model. Nonetheless, following demonstrations can be extended to higher orders.

$$
g(x)=x+0.003 x^{2}-0.005 x^{3} .
$$

The convergence step used is set to $\mu=0.006$. This value, which has been determined in simulations, gives a good compromise between convergence and performance. At the initialization, the estimated polynomial coefficients are set to zero. Due to nonlinear parameters' estimation, the cost function of the NLMS, $J=E\left[\|e(n)\|^{2}\right]$, which is minimized by the algorithm can include several local minima. This is a common issue with this type of algorithm, this makes the choice of the estimated parameters' initial value very important in order to have an optimal solution. Nevertheless, since the considered system is weakly nonlinear, the local minima impact is not very critical.

\section{B. Input distortion level impact on the correction efficiency}

In this subsection, the impact of the input distortion level on the correction performance is studied. As a matter of fact, the correction does not improve the linearity if the input SFDR is smaller than a threshold value. To estimate this point, a normalized two-tone input is used. The considered system can be expressed as:

$$
\begin{gathered}
y(n)=G x(n)+\alpha_{2} x(n)^{2}+\alpha_{3} x(n)^{3} . \\
d(n)=\alpha_{2} x(n)^{2}+\alpha_{3} x(n)^{3}, \\
y_{c}(n)=y(n)-\hat{d}(n)
\end{gathered}
$$

where $d(n)$ is the distortion term, $\hat{d}(n)$ the estimated distortion and $y_{c}$ the corrected signal. To reconstruct the estimated distortion, as said earlier, the following approximation is made: $y(n) \approx G x(n)$.

$$
\hat{d}(n)=\sum_{p=2}^{3} \hat{\alpha}_{p} y^{p}(n) \approx \sum_{p=2}^{3} G^{p} \hat{\alpha}_{p} x(n)^{p} .
$$

Based on the aforementioned approximation, the ideal correction coefficients are $\hat{\alpha_{2}} G^{2}=\alpha_{2}$ and $\hat{\alpha_{3}} G^{3}=\alpha_{3}$. The gain is not of interest in the demonstration, therefore it is fixed to one for convenience. Hence, the corrected output can be developed:

$$
\begin{aligned}
y_{c}= & x-2 \alpha_{2}^{2} x^{3}-\left(\alpha_{2}^{3}+5 \alpha_{2} \alpha_{3}\right) x^{4} \\
& -\left(5 \alpha_{2}^{2} \alpha_{3}+3 \alpha_{3}^{2}\right) x^{5}-\left(\alpha_{2}^{3} \alpha_{3}+7 \alpha_{2} \alpha_{3}^{2}\right) x^{6} \\
& -\left(3 \alpha_{2}^{2} \alpha_{3}^{2}+3 \alpha_{3}^{3}\right) x^{7}-3 \alpha_{2} \alpha_{3}^{3} x^{8}-\alpha_{3}^{4} x^{9} .
\end{aligned}
$$

The considered systems in this study are high performance differential analog front end. As a consequence, the even order harmonics are significantly lower with respect to the odd order harmonics. Hence, the following realistic assumptions are done, $\left|\alpha_{2}\right|<1,\left|\alpha_{3}\right|<1$ and $\alpha_{3}>>\alpha_{2}$. We approximate $y_{c}$ by taking the bigger term in each order:

$$
\begin{array}{r}
y_{c} \approx x-2 \alpha_{2}^{2} x^{3}-5 \alpha_{2} \alpha_{3} x^{4}-3 \alpha_{3}^{2} x^{5}-7 \alpha_{2} \alpha_{3}^{2} x^{6}-3 \alpha_{3}^{2} x^{7} \\
-3 \alpha_{2} \alpha_{3}^{3} x^{8}-\alpha_{3}^{4} x^{9} .
\end{array}
$$

At this point, the input and output SFDR can be compared. The term $-2 \alpha_{2}^{2} x^{3}-3 \alpha_{3}^{2} x^{5}-3 \alpha_{3}^{3} x^{7}-\alpha_{3}^{4} x^{9}$ represents the odd order and $-5 \alpha_{2} \alpha_{3} x^{4}-7 \alpha_{2} \alpha_{3}^{2} x^{6}-3 \alpha_{2} \alpha_{3}^{3} x^{8}$ the even order at the correction output. In order to determine the highest distortion, $x$ is linearized to the power $p$ :

$$
x^{p}=\sum_{i} \alpha_{i_{(p)}} \sin \left(2 p i f_{i_{(p)}} t+\varphi_{i_{(p)}}\right) .
$$

The highest term of distortion for the order $p$ is $\max \left(\alpha_{i_{(p)}}\right)$ with the condition that $f_{i_{(p)}} \neq 0, f_{i_{(p)}} \neq f_{\text {input } 1}$ and $f_{i_{(p)}} \neq$ $f_{\text {input2 }}$. The following table sums up the highest distortion terms for the considered system for order going from 2 to 9 .

\begin{tabular}{l|c|c|c|c}
$\mathrm{p}$ & 2 & 3 & 4 & 5 \\
$\alpha_{i_{(p)}}$ & 0.25 & 0.0938 & 0.1875 & 0.0977 \\
\hline \hline $\mathrm{p}$ & 6 & 7 & 8 & 9 \\
$\alpha_{i_{(p)}}$ & 0.1465 & 0.0897 & 0.1196 & 0.0807
\end{tabular}

The input distortion level which gives the same distortion level at the output is:

$$
-0.0807 \alpha_{3}^{4}-(3 \times 0.0897) \alpha_{3}^{3}-(3 \times 0.0977) \alpha_{3}^{2}=0.0938 \alpha_{3} .
$$

This equation has two complex solutions and one real solution. Since $\alpha_{3}$ must be real, the real solution is kept $\alpha_{3}=-0.5583$. This yields in an input SFDR of:

$$
S F D R_{\text {in }}=10 \cdot \log \left(\frac{P s}{\text { Pdist }}\right)=\underbrace{-15.31}_{P_{s}}-\underbrace{(-31.63)}_{P_{\text {dist }}}=16.32 \mathrm{~dB},
$$

where $P_{s}$ and $P_{d i s t}$ are, respectively, the input and the distortion power.

Figure 2 shows the output SFDR and the SFDR improvement with respect to the input SFDR for a two-tone and a Gaussian noise input signals. The square dot is the 


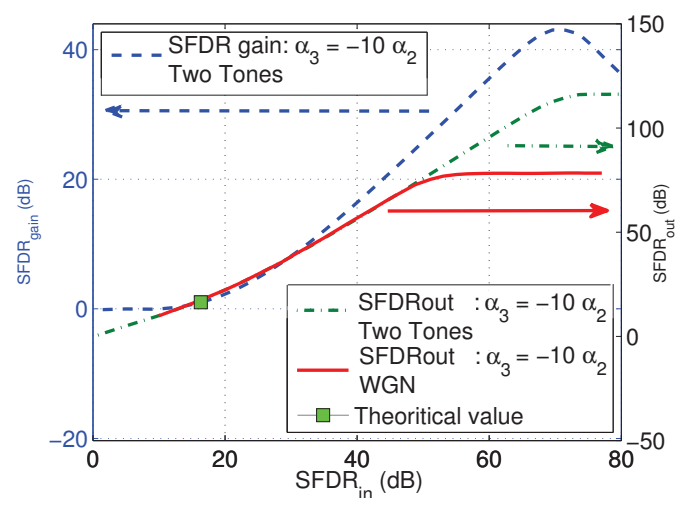

Fig. 2. SFDRin effect: correction limit

theoretical value determined in (10). As can be seen, the SFDR improvement is almost equal to zero when $S F D R_{i n}$ is small. The improvement increases significantly once $S F D R_{i n}$ exceeds the theoretical value. Limitation for high $S F D R_{i n}$ is caused by the ADC noise floor.

\section{Filter Order}

In the employed correction, the purpose of the filter is to retrieve distortions from the free frequency band. As a reminder, this filter is pictured by $f(n)$ in the Fig. 1. Actually, if the adjacent bands are not attenuated enough, the correction efficiency is degraded. The NLMS cost function is $J=E\left[|e(n)|^{2}\right]$. The error signal $e(n)$ is divided into two components $e_{i b}(n)$ and $e_{o b}(n)$ which are, respectively, the error signal in the filter passband and stopband. The NLMS cost function becomes then:

$$
J=E\left[\left|e_{i b}(n)+e_{o b}(n)\right|^{2}\right] .
$$

$e_{i b}(n)$ and $e_{o b}(n)$ are supposed to be independent, therefore $E\left[e_{i b}(n) e_{o b}(n)\right]=0$.

$$
\begin{aligned}
J & =E\left[e_{i b}(n)^{2}+2 e_{i b}(n) e_{o b}(n)+e_{o b}(n)^{2}\right] \\
& =E\left[e_{i b}(n)^{2}\right]+E\left[e_{o b}(n)^{2}\right] .
\end{aligned}
$$

The cost function is then minimized:

$$
\begin{aligned}
\min (J) & =\min \left(E\left[e_{i b}(n)^{2}\right]+E\left[e_{o b}(n)^{2}\right]\right) \\
& \left.=\min \left(E\left[e_{i b}(n)^{2}\right]\right)+\min \left(E\left[e_{o b}(n)^{2}\right]\right)\right) .
\end{aligned}
$$

The above equation corresponds to two NLMS with the following criteria, $\min \left(E\left[e_{i b}(n)^{2}\right]\right)$ and $\left.\min \left(E\left[e_{o b}(n)^{2}\right]\right)\right)$. Let $\hat{e}_{i b}(n)$ be the estimated distortion term with the coefficients retrieved from the first NLMS and $\hat{e}_{o b}(n)$, the estimated distortion term with the coefficients retrieved from the second NLMS. The signal $\hat{e}_{i b}(n)$ has a similar level to the signal distortion in order to cancel it. Likewise, the signal $\hat{e}_{o b}(n)$ has a similar level to the out-band signal. Nonetheless, $\hat{e}_{o b}(n)$ is an error therefore it adds distortions to the corrected signal. In conclusion, if the out-band signal is not weak enough, the correction level is determined by the difference of level between the in-band signal and the adjacent band. Figure 3 shows the effects of the bandpass size and the filter order on the correction. In this simulation, the employed filters are bandpass FIR filter centered around $0.25 \times f s$. A margin of

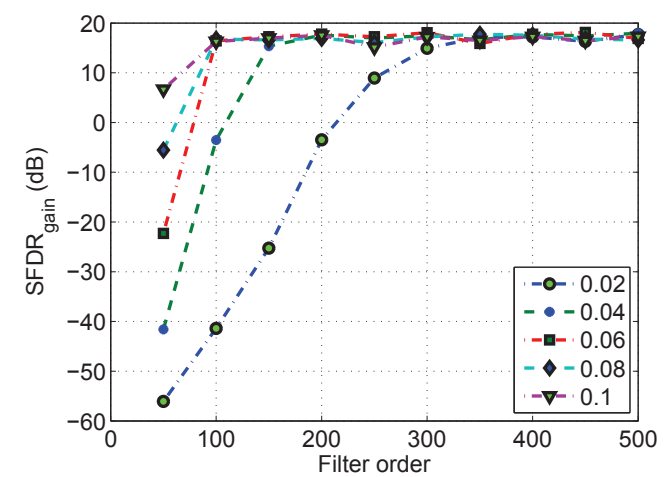

Fig. 3. Filter VS Bandpass analysis

$0.005 \times f s$ on each side of the bandpass is used. The FIR filters are obtained with the Parks-Mclellan algorithm [14]. The choice of the Parks-Mclellan algorithm is motivated by a significantly lower order for a given ideal filter compared to a windowing method. In Fig. 3 , the $S F D R_{\text {gain }}$ is shown with respect to the filter order for different filter bandwidth $F B W$. Small detection band $(B W<0.1)$ are chosen to consider a wideband case. The parameter in the figure is $b w=\frac{F B W}{f s}$. As can be noticed, the smaller the free bandwidth, the higher the filter order required. For practical implementation, the filter order could be reduced if the selected free bandwidth is larger. For example, a $S F D R_{\text {gain }}$ of $15 \mathrm{~dB}$ can be achieved with a filter order less than 100 if $b w>0.1$ Figure 4 shows the input, the output and the corrected output power spectral density. The input is a filtered white Gaussian noise, which is normalized by its amplitude. The filter bandwidth and the filter order were set, respectively, to 0.1 and 100 . The correction is about $15 \mathrm{~dB}$ between the input and the output SFDR.

\section{Quantization}

Another important aspect that should be studied is the signal processing quantization or the employed number of bits after mathematical operation. In fact, a high number of bits reduces the quantization noise but on the other hand increases the complexity and consequently the power consumption as well. Therefore, a good compromise should be found in order to optimize the performance. Two analyzes are carried out to determine this compromise value for the filter coefficients and the calculation wordlength.

Let us first analyze the quantization of the filter coefficients.

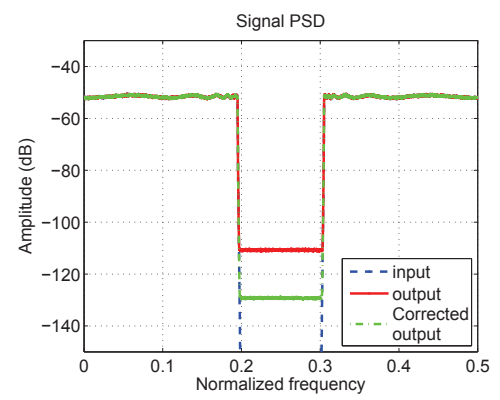

Fig. 4. Signal power spectral density 


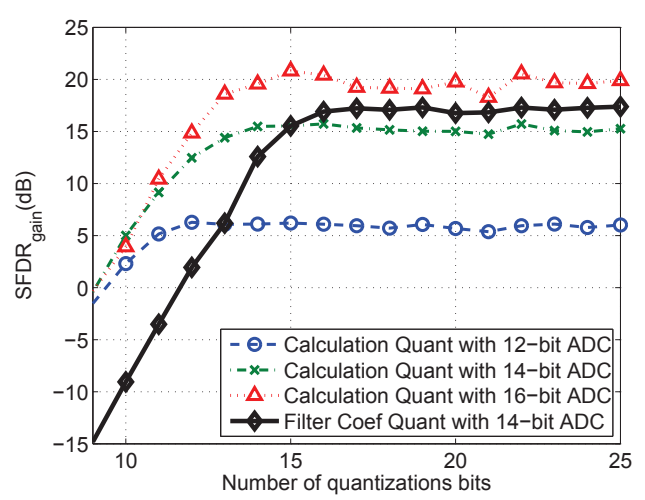

Fig. 5. Quantization effect

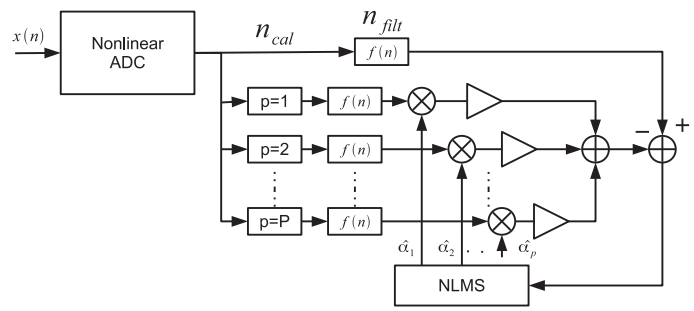

Fig. 6. Quantization scheme

Figure 5 shows the SFDR with respect to the number of bits in four different scenarios. For the four simulations, the input is a white noise filtered with a lowpass filter with a cut-off frequency of $0.3 \times f_{s}$. The thick bold curve shows the impact of quantization on the filters for a 14-bit ADC. As can be seen, the SFDR increases with the number of bits $n_{f i l}$. Actually, the filter out of band attenuation decreases when the coefficients are coded on a lower number of bits. As a consequence, the filtering is less efficient which leads to a SFDR degradation. For the rest of the analysis, the filter coefficients' number of bits is set to 15 which gives a good compromise between complexity and performance. The coefficients are coded using the canonical signed digit (CSD) representation. This representation reduces the number of nonzero bits in the coefficient coding and consequently reduces the filter complexity.

Let us now study the quantization of the signal processing. Figure 6 shows the correction bloc diagram with the quantization at each step of the process. As can be noticed, normalization factors are added when needed to keep the signal in the good range. As a matter of fact, quantization can make the term $\sum_{p=0}^{P}\left\|y^{p}(n)\right\|_{2}^{2}$ become equal to zero which causes divergence. To overcome this problem, one LSB is added to avoid the divergence. Hence, the NLMS expression becomes:

$$
\tilde{\alpha}_{p}(n+1)=\tilde{\alpha}_{p}(n)+\mu \frac{y^{p}(n) e(n)}{\sum_{p=1}^{P}\left\|y^{p}(n)\right\|_{2}^{2}+\epsilon},
$$

where $\epsilon=1 L S B$. Figure 5 shows as well the impact of the calculation quantization for a 12-bit, 14-bit and 16-bit ADC. As expected, the SFDR increases with the number of bits in the 3 scenarios. However, the maximum achievable SFDR is different in each scenario because as said earlier this value depends on the ADC quantization noise floor. For the considered 14-bit ADC, 13 bits of quantization gives a good compromise.

\section{CONCLUSION}

This paper has studied the operation of a post distortion correction for a wideband receiver. The technique uses the signal spreading in the free energy band in order to perform the estimation. Key parameters required for the implementation such as the distortion level, filter complexity and quantization have been analyzed.

The maximum input distortion level has been determined by the means of analytical calculations and confirmed by simulations. Regarding the filters, it has been shown that there is a tradeoff between their complexity (order and number of bits), the size of the free energy band and the linearity improvement.

\section{REFERENCES}

[1] J. Reina-Tosina, M. Allegue-Martinez, M. Madero-Ayora, C. CrespoCadenas, and S. Cruces, "Digital predistortion based on a compressedsensing approach," in European Microwave Conference (EuMC), Oct 2013, pp. 408-411.

[2] D. Schreurs, M. O'Droma, A. A.Goacher, and M. Gadringer, RF Power Amplifier Behavioral Modeling. Cambridge University Press, 2009.

[3] M. Isaksson, D. Wisell, and D. Ronnow, "A comparative analysis of behavioral models for RF power amplifiers," IEEE Transactions on Microwave Theory and Techniques, vol. 54, no. 1, pp. 348-359, Jan 2006.

[4] E. Balestrieri, P. Daponte, and S. Rapuano, "A state of the art on ADC error compensation methods," IEEE Transactions on Instrumentation and Measurement, vol. 54, no. 4, pp. 1388-1394, Aug 2005.

[5] A. Ali, O. Hammi, and T. Al-Naffouri, "Compressed Sensing Based Joint-Compensation of Power Amplifier's Distortions in OFDMA Cognitive Radio Systems," IEEE Journal on Emerging and Selected Topics in Circuits and Systems, vol. 3, no. 4, pp. 508-520, Dec 2013.

[6] N. Kalouptsidis and P. Koukoulas, "Blind identification of VolterraHammerstein systems," IEEE Transactions on Signal Processing, vol. 53, no. 8, pp. 2777-2787, Aug 2005.

[7] P. Cruz and N. Carvalho, "Multi-carrier wideband nonlinear behavioral modeling for cognitive radio receivers," in European Microwave Integrated Circuits Conference (EuMIC), Oct 2011, pp. 414-417.

[8] M. Allen, J. Marttila, and M. Valkama, "Digitally-enhanced wideband analog-digital interfaces for future cognitive radio devices," in 8th IEEE International NEWCAS Conference, June 2010, pp. 361-364.

[9] _ - "Digital post-processing for reducing A/D converter nonlinear distortion in wideband radio receivers," in Conference Record of the Forty-Third Asilomar Conference on Signals, Systems and Computers, Nov 2009, pp. 1111-1114.

[10] L. Duarte, R. Suyama, B. Rivet, R. Attux, J. M. T. Romano, and C. Jutten, "Blind Compensation of Nonlinear Distortions: Application to Source Separation of Post-Nonlinear Mixtures," IEEE Transactions on Signal Processing, vol. 60, no. 11, pp. 5832-5844, Nov 2012.

[11] Y. Ma and Y. Yamao, "Blind nonlinear compensation technique for RF receiver front-end," in European Microwave Integrated Circuits Conference (EuMIC), Oct 2013, pp. 556-559.

[12] K. Shi and A. Redfern, "Blind volterra system linearization with applications to post compensation of ADC nonlinearities," in IEEE International Conference on Acoustics, Speech and Signal Processing (ICASSP), March 2012, pp. 3581-3584.

[13] S. Kalluri and G. Arce, "A general class of nonlinear normalized adaptive filtering algorithms," Signal Processing, IEEE Transactions on, vol. 47, no. 8, pp. 2262-2272, Aug 1999.

[14] J. McClellan and T. Parks, "A unified approach to the design of optimum fir linear-phase digital filters," Circuit Theory, IEEE Transactions on, vol. 20, no. 6, pp. 697-701, November 1973. 